\title{
3D Particle Tracking Velocimetry applied to droplets generated by breaking waves
}

\author{
R.G. Ramirez de la Torre ${ }^{1 *}$, A. Jensen ${ }^{1}$ \\ ${ }^{1}$ University of Oslo Department of Mathematics, Oslo, Norway \\ *reynar@math.uio.no
}

\begin{abstract}
One of the environmental difficulties of exploring the polar regions is marine icing. The understanding of this phenomenon is important for the safety of installations, ships and people that operates in these environments. One of the main sources of marine icing is wave breaking. Therefore, experimental and field work has been conducted to understand the break-up of waves in different situations and some explanation have been proposed to the instabilities that create the spray formation. In this work, two different situations of wave breaking were studied: 1 . Solitary waves were created and steepened by the use of a beach. The waves impacted on a vertical wall with different wall heights. 2. Violent plunging breakers were created by a focusing wave train and a sloping beach. The main objective of these experiments was to quantify the production of droplets from the impact by using Particle Tracking Velocimetry in 3 dimensions. It was found that the initial distribution of droplet sizes is similar in both experiments. These distributions are compared with previous studies, where the distribution of droplet sizes in different experimental cases were approximated by lognormal, Weibull or $\Gamma$-distributions respectively.
\end{abstract}

\section{Introduction}

A large range of two phase flow interactions generate aerosols in the oceanic surface. Between this phenomena, wave breaking is a very important source of aerosol and it is also considered the main source to marine icing (Rashid et al., 2016; Dehghani et al., 2016b; Bodaghkhani et al., 2016), which is the main focus of this work. Marine icing is produced in polar regions, when the droplets produced after wave breaking are transported by the wind and generate thick layers of ice over the surface of ships and structures; these ice-layers represent a life hazard. Therefore, field studies and simulations has been used to address this phenomenon, but its complexity has shown that a deeper understanding of the droplet generation is necessary. To make models of this phenomenon, there is need of more information about the size and velocity distributions of the droplets. It is understood that small aerosols (radius $<1 \mathrm{~mm}$ ) can be transported over long distances and remain in the atmosphere for several days (Veron, 2015). But further studies are necessary to comprehend the generation and dynamics of droplets with radius $>1 \mathrm{~mm}$ because they are relevant to understand the phenomena that occur close to the ocean surface. Recent experimental and field studies (Ortiz-Suslow et al. 2016; Lenain and Melville, 2017) show that the production rates for droplets with radii $\sim 1 \mathrm{~mm}$ were several orders of magnitude higher than the rates expected from previous investigations (Fairall et al., 2009, Veron, 2015). These findings may suggest that large droplets have a longer lifetime in the atmospheric boundary layer than previously expected. Therefore, the processes from where these larger droplets are created need to be better understood. The present study is an attempt to contribute to the understanding of these droplets behaviour. In particular the generation mechanism and initial size distribution.

To measure droplet sizes, optical and non-optical methods can be utilized and some reviews in this topic can be found in the literature (Damaschke et al., 2005; Tayali and Bates, 1990). Non-optical methods rely on either physical separation or impact impressions, which cannot obtain information on the kinematics of the particles. On the other hand, optical techniques rely on imaging or holography, and some of them, like the laser-based measurements, allow the retrieval of velocities simultaneously, therefore these techniques have become popular (Damaschke et al., 2002, Kawaguchi et al., 2002). Nonetheless, some applications are not suitable for the use of laser (Ramirez de la Torre et al., 2020; Løken et al., 2021), hence the use of natural light or scattered light should be reconsidered. In this work, the basis for a scattered-light technique to collect droplet sizes is presented and two different experimental setups are used to test the technique. 

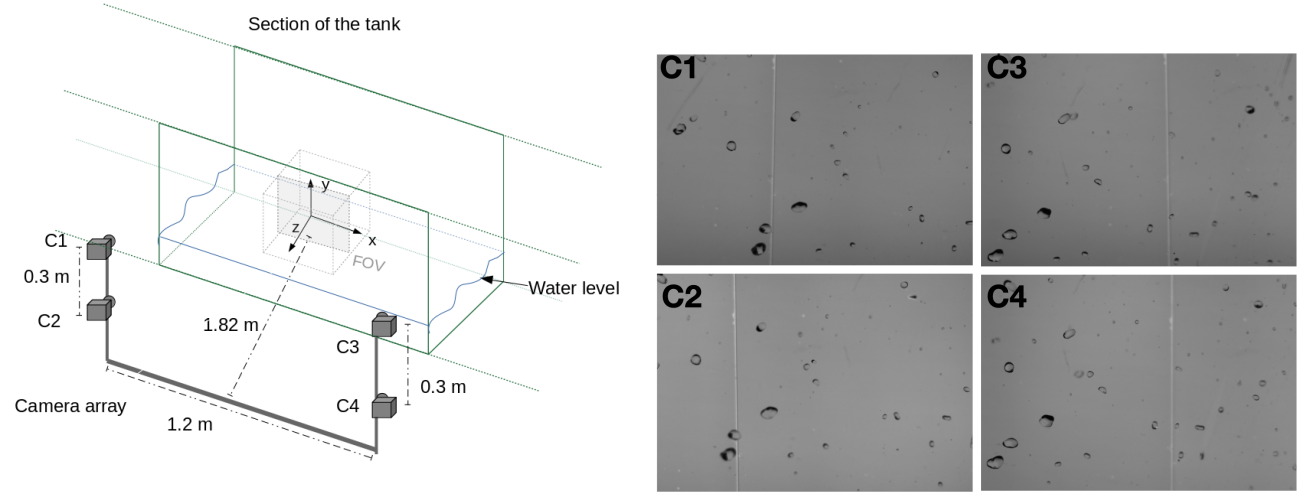

Figure 1: Diagram of the 3DPTV setup in the wave tank. The position of the camera array relative to the tank is shown. An approximation of the FOV and the defined Cartesian system are also shown. On the right side, examples of the images obtained by the camera array are shown.

The jet formation at wave impact and jet break-up into droplets have been less studied because of its complexity. It is common to consider a simplification of the phenomenon by comparing with a planar jet coming out of a nozzle (Lozano et al., 1998; Sarchami et al. 2010; Bodaghkhani et al., 2016) and we can find both numerical and experimental approaches. Only few studies have conducted experimental work on the break up of waves impacting on a vertical wall and proposed an explanation of the instabilities that create the spray formation (Watanabe and Ingram, 2015, 2016). They found that the distribution of droplet sizes at different vertical positions has a lognormal shape and is similar, but not well approximated by distributions proposed by other studies of drop formation. Weibull distribution was proposed by studies of droplet crown formation from a single drop impacting the water surface(Roisman et al., 2006). A $\Gamma$-distribution was proposed for the droplets created after the break-up and coalescence of ligaments that detached from the main water bulk of a circular jet (Villermaux et al., 2004). In the current work, the size distributions of droplets created after the impact of breaking waves is presented. Two significantly different setups are analysed and the results of droplet generation are compared to the previously proposed distributions.

\section{Materials and Methods}

This section will explain the details of two different experimental setups and the technique used to quantify the sizes of the droplets. All experiments were conducted in the wave tank of the Hydrodynamics Laboratory at the University of Oslo. The wave tank has dimensions $25 \times 0.52 \times 1 \mathrm{~m}$. In the first set of experiments, solitary waves impacting on a vertical wall were analysed. Different wave heights were used to obtain different breaking stages and the wall height is lower than the wave height in all cases. In the second set of experiments, a focusing wave train was forced to overturn by means of a beach. Three different amplitudes of the focusing wave train were used and two different wind speeds were also imposed on the air phase. The main objective of these experiments was to quantify the production of droplets from the impact and relate it to the impact characteristics. In contrast to other investigations, the droplets were measured using three dimensional Particle Tracking Velocimetry (3DPTV) and shadowgraphy images.

\subsection{DPTV to obtain droplet sizes}

We analyze all the droplets that were visible in the selected field of view (FOV) of each experiment, using the setup presented in figure 1 . The analysis was made by means of 3DPTV with the open source program OpenPTV Consortium et al. (2012). Highly resolved, high cadence (167 fps) images of the cloud are taken by 4 Monochromatic AOS Promon cameras with $50 \mathrm{~mm}$ lenses, examples of the obtained images are shown also in figure 1. LED lamps and white diffusive sheets were used to illuminate the back of the setup. The position of the FOV is always after the impact location and depends on the experimental case. A sequence of 2 seconds during and after the impact is recorded.

The droplet size detection was made by combining the trajectories found by 3DPTV and additional post processing. In which the pixel size of each detected particle is found and an estimation of its diameter 


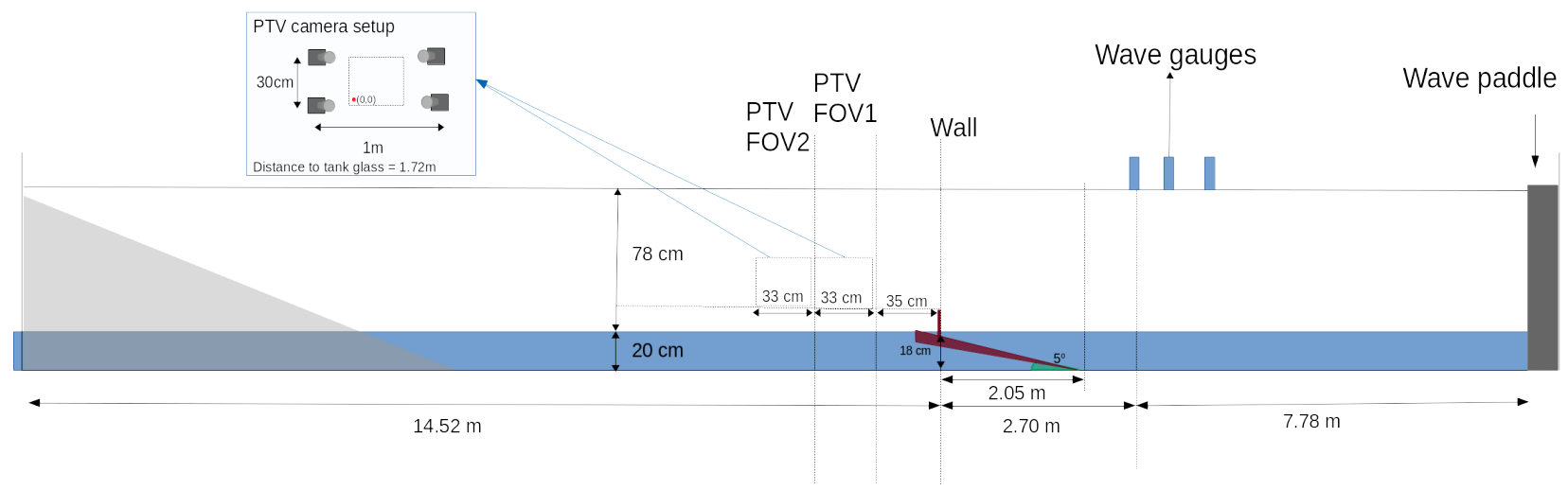

Figure 2: Diagram of the experimental setup in the wave tank. The position of the beach and the wall is shown. Also the camera array and distances to the glass wall are shown. Two different FOV's are used to visualize the droplets. The camera array is moved to visualize each FOV separately .

is made by means of previously established relations. The process is summarized in the following: With the PTV algorithm we define a position of each detected droplet in the three-dimensional space and follow it through time $s(t)=(x, y, z)$. Previously to the measurements, a calibration of the system needs to be performed in which relations between all the cameras FOV and the Cartesian space are established. This calibration can be used to also establish a pixel-to-world transform for each camera $i=1,2,3,4$ and a correction due to the light scattering around the droplets depending on the depth of the particle $T(i, z)$. Once $s(t)$ is calculated by PTV, the pixel size of the droplet in the vertical and horizontal direction $p_{x}(s), p_{y}(s)$ can be obtained from the data files of each camera $i$. Then, an estimation of the droplet axes $a, b$ in each camera can be done by applying the defined transformation $a_{i}(s)=T\left(i, z(s) ; p_{x}\right)$ and $b_{i}(s)=T\left(i, z(s) ; p_{y}\right)$. It is important to mention that droplets are not always spherical and their deformation increases with the size. The equivalent diameter $D_{e}$ is commonly used to classify droplet sizes with one unique parameter and is commonly defined as $D_{e}=\sqrt{a b}$, where $a$ and $b$ are the major and minor axis of the ellipsoid. In addition, $a=\overline{a_{i}}$ and $b=\overline{b_{i}}$, the averaged values from the 4 cameras.

To obtain size distributions, we collect all the detected droplets in every time step during the time series and throughout all the repetitions of the experiments. Ten repetitions of the same case were developed for the first experiment, while only 5 repetitions were needed for the second experiment. In this way, we obtain one distribution for each analyzed parameter. In other words, we obtain one distribution for each amplitude and wall height in the first experiment, and one distribution for each steepness and wind velocity in the second experiment.

\subsection{Experiment 1: Solitary wave impacts in a vertical wall}

In the first set of experiments, the mean water level for all experiments was $d=0.2 \mathrm{~m}$. The aim of this work was to generate steep solitary waves which will impact in a wall at different stages of the breaking process. To achieve this, solitary waves were made by a horizontal displacement wave paddle, shown in figure 2. A PETG (Polyethylene Terephthalate Glycol-Modified) beach with an inclination of $5^{\circ}$ was placed at $8.43 \mathrm{~m}$ from the rest position of the paddle. A vertical wall was positioned at $2.05 \mathrm{~m}$ from the vertex of the beach. The amplitude $(A)$ to depth $(d)$ ratio, or non dimensional amplitude $a=A / d$ was varied as $a=0.5,0.46,0.4,0.35$. Two different wall heights $h$ were used for each $A$, with an amplitude to wall ratio $h / A \approx 0.8,0.9$. The produced droplets are observed in two FOV's, after the impact area (marked as FOV1 and FOV2 in fig. 2).

To determine the breaking stage of the input wave, simulations were made with a Boundary Integral Method (BIM) Pedersen (2008). The wave amplitude was measured with 3 acoustic wave gauges between the beach and the wave paddle. The gauges were positioned at $7.07 \mathrm{~m}, 7.42 \mathrm{~m}$ and $7.78 \mathrm{~m}$ from the rest position of the paddle respectively. Comparison of the simulations and the gauge measurements was done. Far from the wall, the simulations and the experiments are in good agreement. The used BIM model cannot simulate the trapped air cavity between the tip and the trough. This is because the simulation cannot handle two contact points between the surface and the boundary. Therefore, the velocities in the trough cannot be 

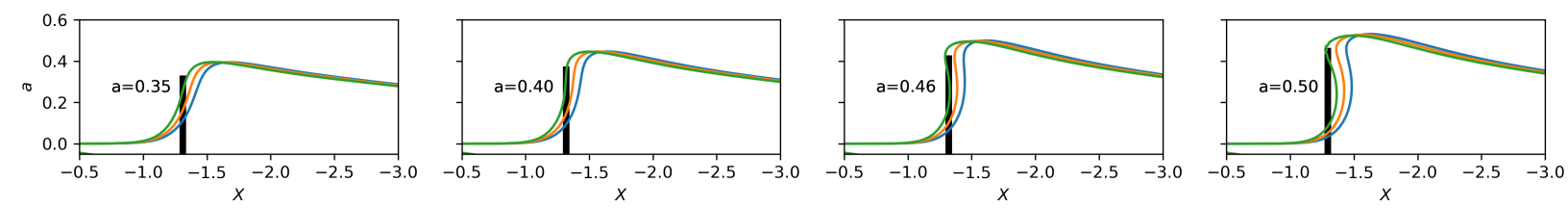

Figure 3: Results of the BIM model simulation for the breaking stages of the different wave amplitudes $a$. The wall is represented by the black line. Three time steps of the simulations are presented, when the wave is close to the wall position.

approximated. In spite of this, there is similarity in the shape and speed of the wave tip approaching the wall until before impacting the wall. From these simulations we can approximate the breaking point and size of the air cavity between the wall and the wave. Fast imaging was used to visualize the impact, from the images three different impacts can be distinguished: flip-through $(a=0.35)$ without air pocket, small air pocket $(a=0.40)$ and big air pocket $(a=0.46,0.50)$. With this images we confirm the the simulations are a good approximation to the wall position.
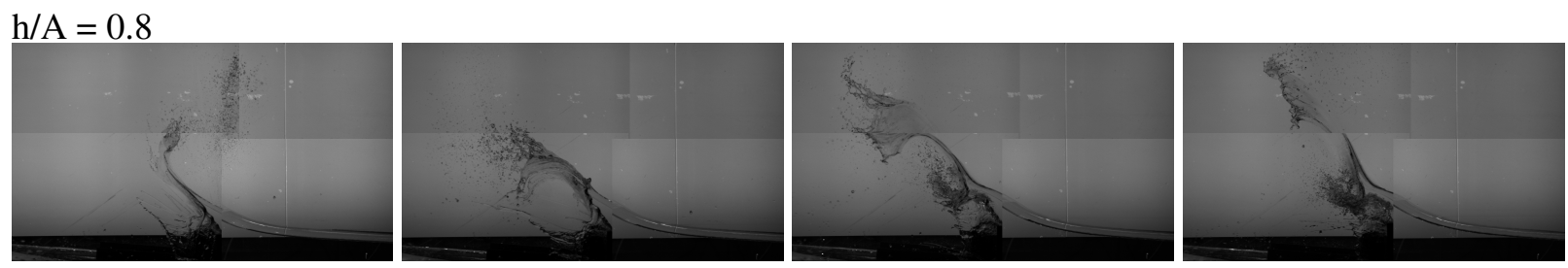

$\mathrm{h} / \mathrm{A}=0.9$
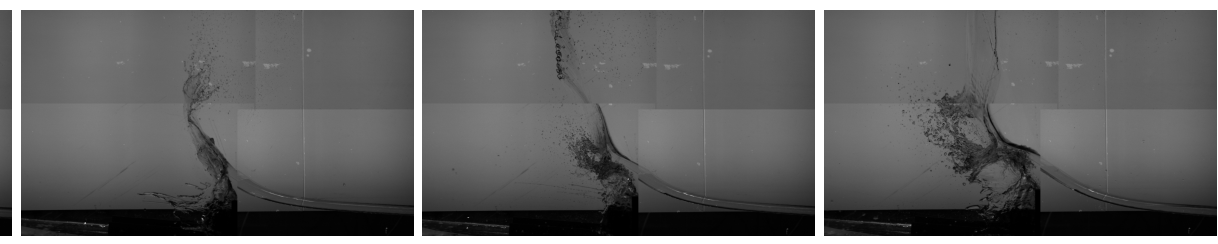

Figure 4: Splashing of a wave into the wall, the initial moments of the jet breaking into droplets are visualized in all cases. From the left: $a=0.35,0.40,0.46,0.50$. The top row shows the cases with wall of height $h / A=0.8$ and to the bottom row, the cases with wall height $h / A=0.9$..

Figure 4 shows the different impacts obtained in the experiment. After the impact, all cases form a planar jet that shoots upwards in a certain angle. For every impact type we have also two different wall heights that result in a different releasing angle of the planar jet. On top of that, for the cases where there is an air pocket, a second jet is visible when the air cavity is closed, each of these jets has a different ejection angle. The aim of these images is to show the development of the wave after the impact. It is interesting to observe the differences arisen by the different amplitudes and wall heights. The formation of the first jet and the time and angle were the air pockets closed have noticeable changes.

\subsection{Experiment 2: Focusing wave train (FWT) and wind conditions}

In the second set of experiments, a focusing wave train (FWT, for short) was used to produce breaking waves, the mean water level was $d=0.5 \mathrm{~m}$. In the wave train, long waves overtake short waves, then the breaking was made more violent by adding a slope which caused the already focused waves to steepen and overturn. The overturning crest of the wave splashed at the free surface releasing a large number of droplets. Further details of this set of experiments can be found in Ramirez de la Torre, Vollestad, and Jensen (2020).

The wave trains were created using the same methodology as presented in Brown and Jensen (2001). By using this focusing method, we obtain breaking waves when we reach steepness $a k>0.44$. But the breaking created by the selected amplitudes only generated spilling breakers and small overturning. Therefore, a shoaling was added to steepen the waves even more as they approach the focusing point. In this way, 


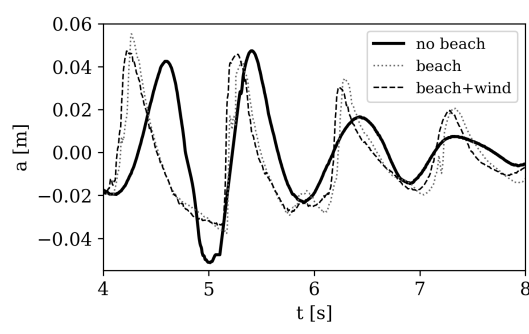

(a) Surface elevation

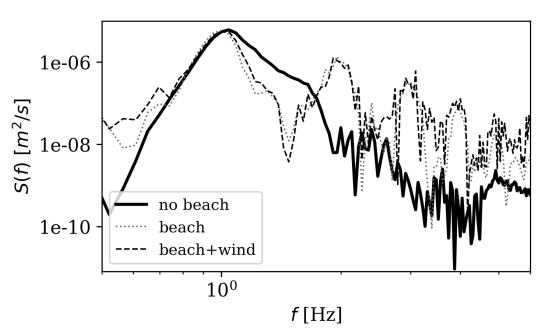

(b) Power spectrum

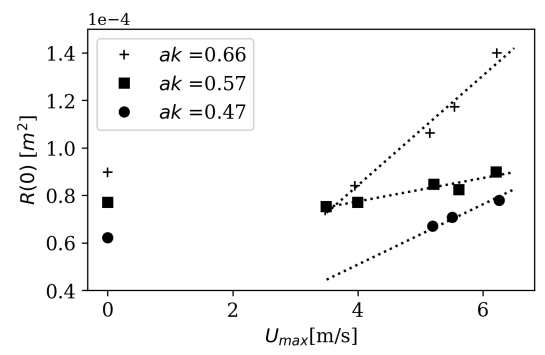

(c) Energy content

Figure 5: Surface elevation (a) and Power Spectrum (b) at the focal point for cases without beach ("no beach" label, solid line), with beach and without wind ("beach" label, dotted line), and with beach and wind ("beach+wind" label, dashed line). $a k=0.57$ has been selected as example and $U_{\max }=6.2 \mathrm{~ms}^{-1}$ in the wind case. (c) shows mean power $R(0)$ compared to $U_{\max }$ for the three $a k$, which represented by different markers, the graph shows the increase of energy content with both wind speed and steepness. Images extracted from Ramirez de la Torre, Vollestad, and Jensen (2020).

the waves are forced to overturn. The steepness $a k$ can be used as non dimensional parameter to identify the different wave trains, which are $a k=0.47,0.57,0.66$. The wind profiles, without the influence of mechanically generated waves, were measured using particle image velocimetry (PIV). The peak horizontal velocity recorded $\left(U_{\max }\right)$ is correlated to the pressure change $(p)$ obtained by a pressure gauge, and this correlation is used to approximate the mean velocity of the wind during the experiments with the FWT. The wind velocities used in the experiments were $U_{\max }=0,5.2$ and $6.2 \mathrm{~ms}^{-1}$

Figure 5 summarizes the results of introducing a beach and wind forcing to the FWT. By comparing the surface elevation for the FWT with and without beach (figure 5(a)), it is visible that there is a steepening effect in the beach cases. This steeper central high component produces a violent plunger breaker that can be studied. The energy content of the wave group can be quantified by means of the power spectrum $S(f)$, shown in figure 5(b). It is obvious that all cases have the same peak frequency, but the beach cases show evidence of energy dispersion. To quantify the change in energy content of the different cases, we can use the mean power $R(0)$, defined as the area under the spectral curve $S(f)$, which can be interpreted as the energy content of the wave as $R(0) \propto a^{2}$ which is also proportional to the energy. Figure 5(c) shows the calculated $R(0)$ compared to the different maximum wave steepness: $a k$ and wind velocities: $U_{\max }$ used for this work. The graph shows the effect of wind over the wave energy. In all cases the energy increases with $a k$. But, it is interesting to see that for $U_{\max }<4.5$ the total energy of the packet is less than the energy of the packet without the presence of wind.

\section{Results}

Figure 6 shows the probability distributions of equivalent diameter $D_{e}$ for different cases. All distributions are normalized by the mean $\overline{D_{e}}$. In figure $6(\mathrm{a})$, the results of experiment 1 , with the solitary waves, are presented, and in figure 6(b) the results of experiment 2, with the FWT, are presented. In both cases the distribution has a similar shape, with and extended tail towards larger sizes and a unique maxima that is not centered. For each experiment, the change of parameter shows a displacement of the tail and the maxima. A discussion on the theoretical distributions that can fit the data will be done, but first we will analyze the results for the mean diameter, $\overline{D_{e}}$, of each case.

Figure 7 shows the change in $\overline{D_{e}}$ as a function of the parameters determined for each experiment. In the first experiment (fig. 7(a)), two parameters were used: the non dimensional amplitude $a=A / d$ (fig. 7(a) left), and the non dimensional jet speed defined as $v_{\text {jet }} / c$ (fig. 7(a)-right), where $c$ is the wave speed, obtained by solitary wave theory $(c=\sqrt{(d+A) / d})$ and the jet speed $v_{\text {jet }}$ can be estimated by the displacement of the jet in the images divided by the time elapsed between them. For $D_{e}$ vs $a$, a slight peak is visible when $a=0.40$ and the values for FOV1 are always larger. This is expected as in FOV2, the droplets have spend more time flying and further break-up is expected, which corresponds to smaller sizes. For $D_{e}$ vs $v_{j e t} / c$, we see a more defined trend where, the mean size of the droplet decreases for $v_{j e t} / c>2$. Dehghani et al. 


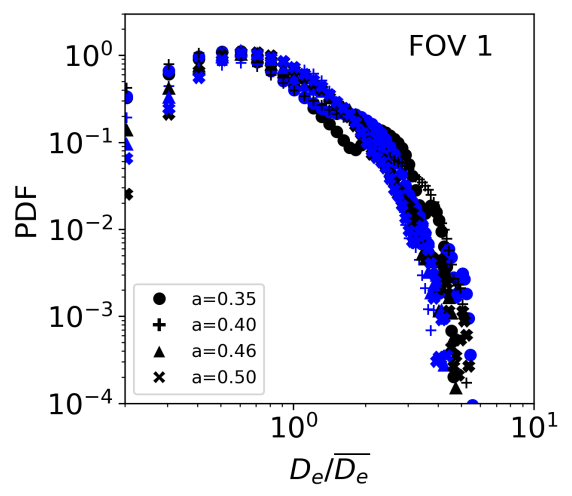

(a) Experiment 1

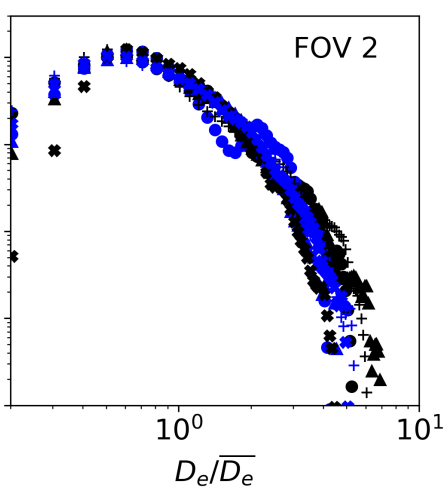
,

Figure 6: Probability Distribution Function of $D_{e}$ normalized by the mean for the different experiments. (a) shows the results for Experiment 1, with the solitary waves. To the left, the results for FOV1 are shown and to the right, the results for FOV2 are shown. The different colors describe the wall height: blue for $h / A=0.9$ and black $h / A=0.8$, the markers show the different $a$ as shown in the graph label. (b) shows the results of Experiment 2, with the FWT, the different colors represent the different wind conditions: black for $U_{\max }=0$, blue for $U_{\max }=5.2$ and red for $U_{\max }=6.2$. The different markers shows the different steepness as presented in the label.

(2016a) have predicted a similar change of size in the droplets, for larger velocities in the jet, finer droplets are expected. It is also interesting to see that for the cases where $v_{j e t} / c<2$ the mean droplet size decreases, which suggest that the relation between speed and size is not linear and that the ratio of the jet speed and the wave speed could be a better parameter to represent the sizes on wave impact, but further data would be necessary to test this hypothesis.

In the second experiment (fig. 7(b),$R(0)$ is used as a parameter that quantifies the wave energy. It is observed that $\overline{D_{e}}$ increases with $R(0)$ of the wave. Previously, it was found that the mean size of droplets decreases with the presence of high winds Mueller and Veron (2009); Ortiz-Suslow et al. (2016); Fairall et al. (2009). Our findings suggest that the energy of the waves while breaking is also an important parameter on the size distribution and should be considered together with the wind velocity.

As for the distribution of droplet sizes in Figure 8, three different models from the literature, were used to compare to the data (Villermaux et al., 2004; Watanabe and Ingram, 2016; Roisman et al., 2006). Without loss of generality, in figure 8 , an example case has been selected from each experiment and the three models

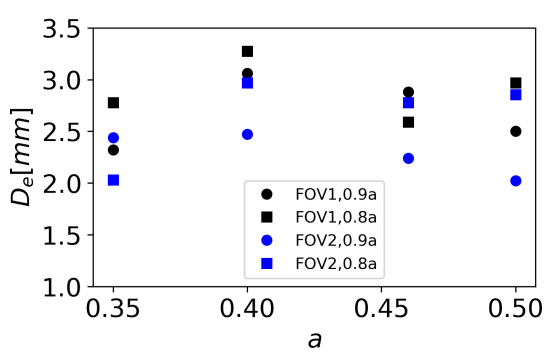

(a) Experiment 1

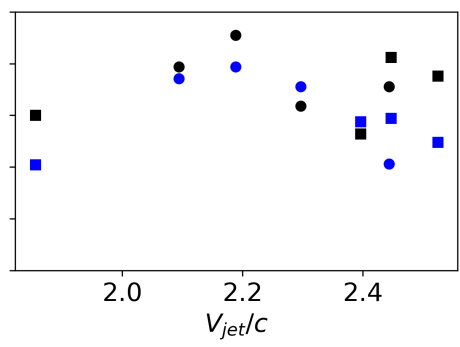

Figure 7: $D_{e}$ against the experimental parameters, (a) shows the results of Experiment 1, two parameters are defined: non-dimensional amplitude $a$ and non dimensional jet velocity $v_{\text {jet }} / c$ ( $c$ : wave speed). Black represents FOV 1 and blue for FOV2, squares represents $h / A=0.8$ and circles represents $h / A=0.9$. (b) shows the result for Experiment 2 where the main parameter is $R(0)$ an estimate of the wave packet energy. It is important to remember that $R(0)$ depends on $U_{\max }$ and $a k$, as shown in figure 5 . 


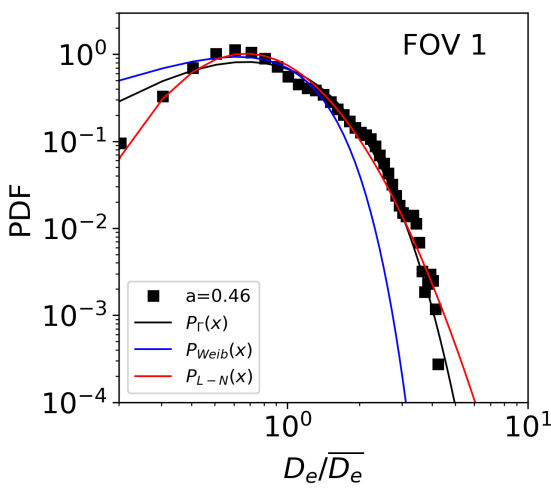

(a) Experiment 1
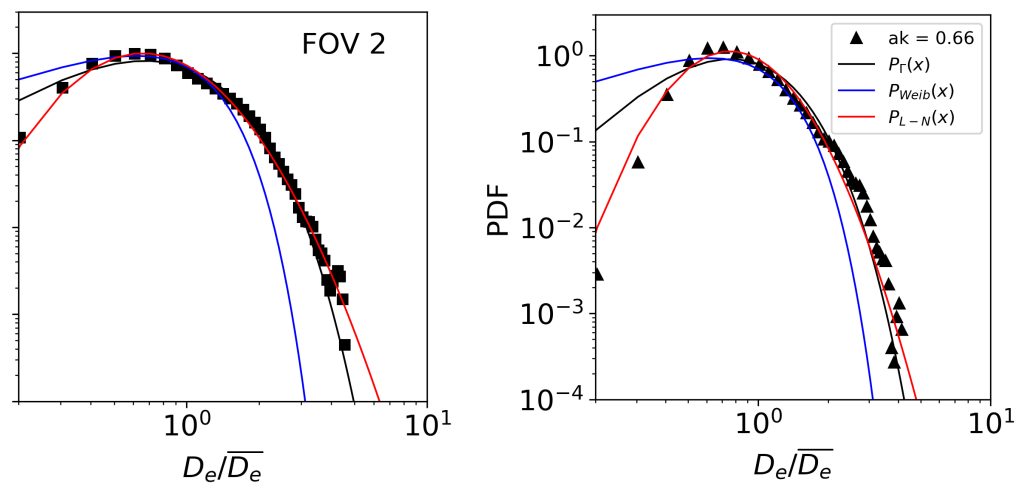

(b) Experiment 2

Figure 8: Probability Distribution Function of $D_{e} / \overline{D_{e}}$ compared to theory models. The squares shows the experimental data and the colored lines represent the different models proposed by the references: black for Villermaux et al. (2004), blue for Roisman et al. (2006) and red for Watanabe and Ingram (2016). (a) shows results of Experiment 1 for the case of $a=0,46, h / A=0.8$, results for FOVI are presented to the left while results for FOV2 are presented to the right. (b) shows results for the FWT case, $a k=0.66$ and $U_{\max }=6.2 \mathrm{~ms}^{-1}$ are the parameters in the chosen data set.

are fitted to the data. The first model, was presented by Villermaux et al. (2004) (black line):

$$
P_{\Gamma}(x ; n)=\frac{n^{n} x^{n-1} e^{-n x}}{\Gamma(n)}
$$

where $n^{-1}$ is the variance and $x=D_{e} / \overline{D_{e}}$ is the diameter normalized by the mean. This model describes the ligament-mediated spray formation and was originally thought for a jet of water that creates ligaments and droplets by the effect of wind shear in the surface of the jet. The second model was presented by Roisman et al. (2006) (blue line):

$$
P_{W e i b}(x ; \alpha, \beta)=\frac{\beta}{\alpha}\left(\frac{x}{\alpha}\right)^{\beta-1} e^{-\left(\frac{x}{\alpha}\right)^{\beta}}
$$

where $\alpha=0.89$ and $\beta=1.94$ are empirically found. This model was used to describe the secondary droplets created by the rim instability in crown splashes. The third model was presented in Watanabe and Ingram (2016) (red line):

$$
P_{L-N}(x ; \mu, \lambda)=\frac{1}{x \lambda \sqrt{2 \pi}} e^{\frac{-(\ln x-\mu)^{2}}{2 \lambda^{2}}},
$$

where $\mu=\overline{\ln x}$ is the mean of the natural logarithm of the normalized size and $\lambda=\operatorname{std}[\ln x]$ is the standard deviation. This model was used to describe the generation of droplets from impacting waves on a vertical wall.

From figure 8 , it is visible that only $P_{\Gamma}$ and $P_{L-N}$ follow closely the data for values larger than the mean, which is the maximum of the distribution, and that only $P_{L-N}$ follows the data more accurately for values smaller than the mean. The experimental data for droplet sizes below $0.5 \mathrm{~mm}$ has a larger error because of the cameras resolution, therefore it is more significant to concentrate on the droplets with sizes larger than $0.5 \mathrm{~mm}$. In this case, only $P_{\Gamma}$ seems to resemble closely the distribution without overestimating the probability. A quantitative manner to analyze the closeness of the distribution to the experimental data is to calculate the theoretical quantiles for the different models and compare them to the quantiles of the experimental data, if the model is a good fit the quantiles should align in the identity line $Q(\exp )=Q($ theory). Over all, the first model that corresponds to Villermaux et al. (2004), shows the closest similarity for all the quantile values. From this analysis we can conclude that $P_{\Gamma}$ shows the best fit for the data presented in this study. $P_{L-N}$ provides a good fit for the lowest section of the quantiles but separates quite much in the largest values, while $P_{W e i b}$ shows big difference in the extreme values. 


\section{Acknowledgements}

Funding from the Norwegian Research Council through the project 'Rigspray' (grant number 256435) is gratefully acknowledged. The authors will also like to acknowledge Alex Liberzon and the OpenPTV Software Consortium for the help with the use of OpenPTV. The help of Olav Gundersen in the experimental setup is gratefully acknowledge.

\section{References}

Bodaghkhani A, Dehghani SR, Muzychka YS, and Colbourne B (2016) Understanding spray cloud formation by wave impact on marine objects. Cold Regions Science and Technology 129:114-136

Brown MG and Jensen A (2001) Experiments on focusing unidirectional water waves. Journal of Geophysical Research: Oceans 106:16917-16928

Consortium O et al. (2012) Openptv: Open source particle tracking velocimetry. 2012,()

Damaschke N, Nobach H, Nonn TI, Semidetnov N, and Tropea C (2005) Multi-dimensional particle sizing techniques. Experiments in fluids 39:336-350

Damaschke N, Nobach H, and Tropea C (2002) Optical limits of particle concentration for multidimensional particle sizing techniques in fluid mechanics. Experiments in fluids 32:143-152

Dehghani S, Muzychka Y, and Naterer G (2016a) Droplet trajectories of wave-impact sea spray on a marine vessel. Cold Regions Science and Technology 127:1-9

Dehghani S, Naterer G, and Muzychka Y (2016b) Droplet size and velocity distributions of wave-impact sea spray over a marine vessel. Cold Regions Science and Technology 132:60-67

Fairall C, Banner M, Peirson W, Asher W, and Morison R (2009) Investigation of the physical scaling of sea spray spume droplet production. Journal of Geophysical Research: Oceans 114

Kawaguchi T, Akasaka Y, and Maeda M (2002) Size measurements of droplets and bubbles by advanced interferometric laser imaging technique. Measurement Science and Technology 13:308

Lenain L and Melville WK (2017) Evidence of sea-state dependence of aerosol concentration in the marine atmospheric boundary layer. Journal of Physical Oceanography 47:69-84

Løken TK, Ellevold TJ, de la Torre RGR, Rabault J, and Jensen A (2021) Bringing optical fluid motion analysis to the field: a methodology using an open source ROV as a camera system and rising bubbles as tracers. Measurement Science and Technology 32:095302

Lozano A, Garcia-Olivares A, and Dopazo C (1998) The instability growth leading to a liquid sheet breakup. Physics of fluids 10:2188-2197

Mueller JA and Veron F (2009) A sea state-dependent spume generation function. Journal of physical oceanography 39:2363-2372

Ortiz-Suslow DG, Haus BK, Mehta S, and Laxague NJ (2016) Sea spray generation in very high winds. Journal of the Atmospheric Sciences 73:3975-3995

Pedersen G (2008) A lagrangian model applied to runup problems. Advanced numerical models for simulating tsunami waves and runup 10:311-314

Ramirez de la Torre R, Kuchta M, and Jensen A (2020) Experiments on air entrainment produced by a circular free falling jet. International Journal of Multiphase Flow 132:103424

Ramirez de la Torre RG, Vollestad P, and Jensen A (2020) Experimental investigation of droplet distributions from a plunging breaker with different wind conditions. Earth and Space Science Open Archive page 17

Rashid T, Khawaja HA, and Edvardsen K (2016) Review of marine icing and anti-/de-icing systems. Journal of Marine Engineering \& Technology 15:79-87 
Roisman IV, Horvat K, and Tropea C (2006) Spray impact: rim transverse instability initiating fingering and splash, and description of a secondary spray. Physics of Fluids 18:102104

Sarchami A, Ashgriz N, and Tran H (2010) An atomization model for splash plate nozzles. AIChE journal 56:849-857

Tayali N and Bates C (1990) Particle sizing techniques in multiphase flows: a review. Flow Measurement and Instrumentation 1:77-105

Veron F (2015) Ocean spray. Annual Review of Fluid Mechanics 47:507-538

Villermaux E, Marmottant P, and Duplat J (2004) Ligament-mediated spray formation. Physical review letters 92:074501

Watanabe Y and Ingram D (2015) Transverse instabilities of ascending planar jets formed by wave impacts on vertical walls. Proceedings of the Royal Society A: Mathematical, Physical and Engineering Sciences 471:20150397

Watanabe Y and Ingram D (2016) Size distributions of sprays produced by violent wave impacts on vertical sea walls. Proceedings of the Royal Society A: Mathematical, Physical and Engineering Sciences 472:20160423 